\title{
Correction to: Improved precision in the analysis of randomized trials with survival outcomes, without assuming proportional hazards
}

\author{
Iván Díaz ${ }^{1}$ - Elizabeth Colantuoni ${ }^{2}$ - Daniel F. Hanley ${ }^{3} \cdot$ Michael Rosenblum $^{2}$ \\ Published online: 4 September 2019 \\ ○) Springer Science+Business Media, LLC, part of Springer Nature 2019
}

\section{Correction to: Lifetime Data Anal (2019) 25:439-468 https://doi.org/ 10.1007/s10985-018-9428-5}

The original version of this article unfortunately contains mistakes. It has been corrected with this Correction.

\section{Summary}

The $\mathrm{R}$ code used for the data analysis and simulations in our manuscript (Díaz et al. 2018) had two errors, which we have corrected. All of the theoretical results in the paper are correct. It is our implementation of these in R code that had the errors, which impacts the data analysis and simulation results in Section 6. This erratum describes the errors and presents the updated results of the data analysis and simulations with the errors corrected.

The two errors were (i) incorrect coding of the auxiliary variable $H$ used in the TMLE estimator, and (ii) incorrect coding of time $t$ as numeric instead of as a factor in the adjusted estimators. These were corrected and the updated code is available at (Díaz 2018a). The updated results, given below, are qualitatively similar to the original results (i.e., there are no changes to our conclusions in the paper) except for the following: the updated TMLE $\left(\hat{\theta}_{\text {adj,eff }}\right)$ confidence interval coverage probabilities ranged from 93 to $95 \%$ (previously 94-95\%); the updated bias of the TMLE $\hat{\theta}_{\text {adj,eff }}$

The original article can be found online at https://doi.org/10.1007/s10985-018-9428-5.

$凶 \quad$ Iván Díaz

ild2005@med.cornell.edu

1 Division of Biostatistics and Epidemiology, Weill Cornell Medicine, New York, NY, USA

2 Department of Biostatistics, Johns Hopkins Bloomberg School of Public Health, Baltimore, MD, USA

3 Division of Brain Injury Outcomes, Johns Hopkins Medical Institutions, Baltimore, MD, USA 
Table 1 Estimated RMST (standard error) with $95 \%$ bias-corrected and accelerated (BCa) bootstrap confidence intervals based on the CLEAR III trial

\begin{tabular}{lll}
\hline Estimator & RMST (SE) & $95 \%$ BCa confidence interval \\
\hline KM & $14.9(5.3)$ & $(5.6,26.2)$ \\
IPW & $14.9(5.3)$ & $(5.6,26.2)$ \\
Adj. IPW & $15.5(5.6)$ & $(6.0,27.9)$ \\
AIPW & $14.5(5.2)$ & $(4.9,25.1)$ \\
TMLE & $14.4(5.0)$ & $(5.4,24.7)$ \\
\hline
\end{tabular}

was sometimes larger but still small (at most 3\%) as a fraction of the treatment effect (14.9 days) and had negligible influence on the mean squared error; the adjusted inverse probability weighted estimator $\hat{\theta}_{\text {adj,ipw }}$ (which was not the focus of the paper) had larger variance in the updated results compared to the original results, leading to lower relative efficiency.

\section{Corrections to $\mathbf{R}$ code}

First, the $\mathrm{R}$ code for computing the auxiliary variable $H(m, A, W)$ involved in the

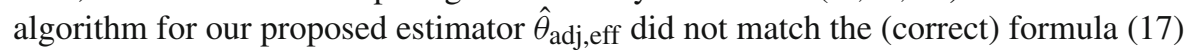
from the paper. We have fixed this error in the R package (Díaz 2018a). Please refer to the GitHub commit given in that citation at the end of this erratum for the specific lines of code that were corrected.

Second, when computing all adjusted estimators, including $\hat{\theta}_{\text {adj,ipw }}$ and $\hat{\theta}_{\text {adj,eff, the }}$ time $t$ should have entered our R code (in the working models for $g_{A}, g_{R}$ ) as a variable of type factor rather than as numeric (see Díaz 2018b). This is needed in order to satisfy the requirement in Theorem 2 that dropout probabilities are estimated using models with saturated terms for time, treatment, and their interaction.

We have fixed the above two errors and reported the results of our revised analyses in Tables 1 through 3, whose numbering corresponds to the labels of tables in the original paper. Corresponding to our fix of the second error, the sentence "The model for $g_{R}$ for the adjusted estimators includes main terms for time (linear), treatment and their interaction, in addition to main terms for $W_{1}$ and $W_{5}$ and a treatment by $W_{3}$ interaction." from Section 6.3 should now have "(linear)" replaced by "(factor)".

\section{CLEAR III trial data analysis}

The results of analyzing the CLEAR III trial data were qualitatively similar to our initial findings. The Kaplan-Meier estimate of the RMST is 14.9 days (SE 5.3, 95\% BCa CI 5.6-26.2). Our TMLE estimator $\hat{\theta}_{\text {adj, eff has an estimated variance that is roughly }}$ $13 \%$ smaller than the (unadjusted) RMST difference based on the cMeier estimator (compared to $16 \%$ smaller as reported in the original manuscript).

\section{Simulation studies}

Tables 2 and 3 display the new simulation results. The updated results were qualitatively similar to the original results, except for the following: the updated TMLE 


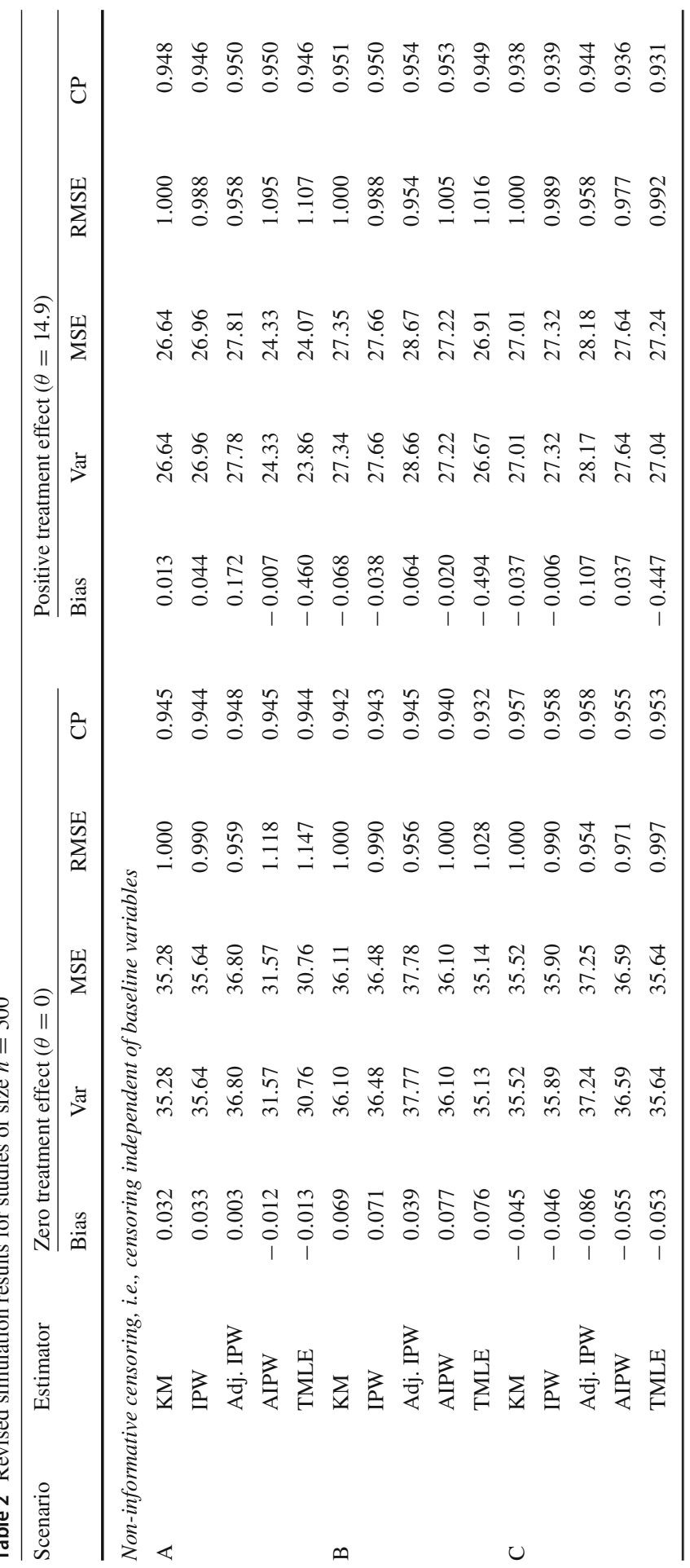




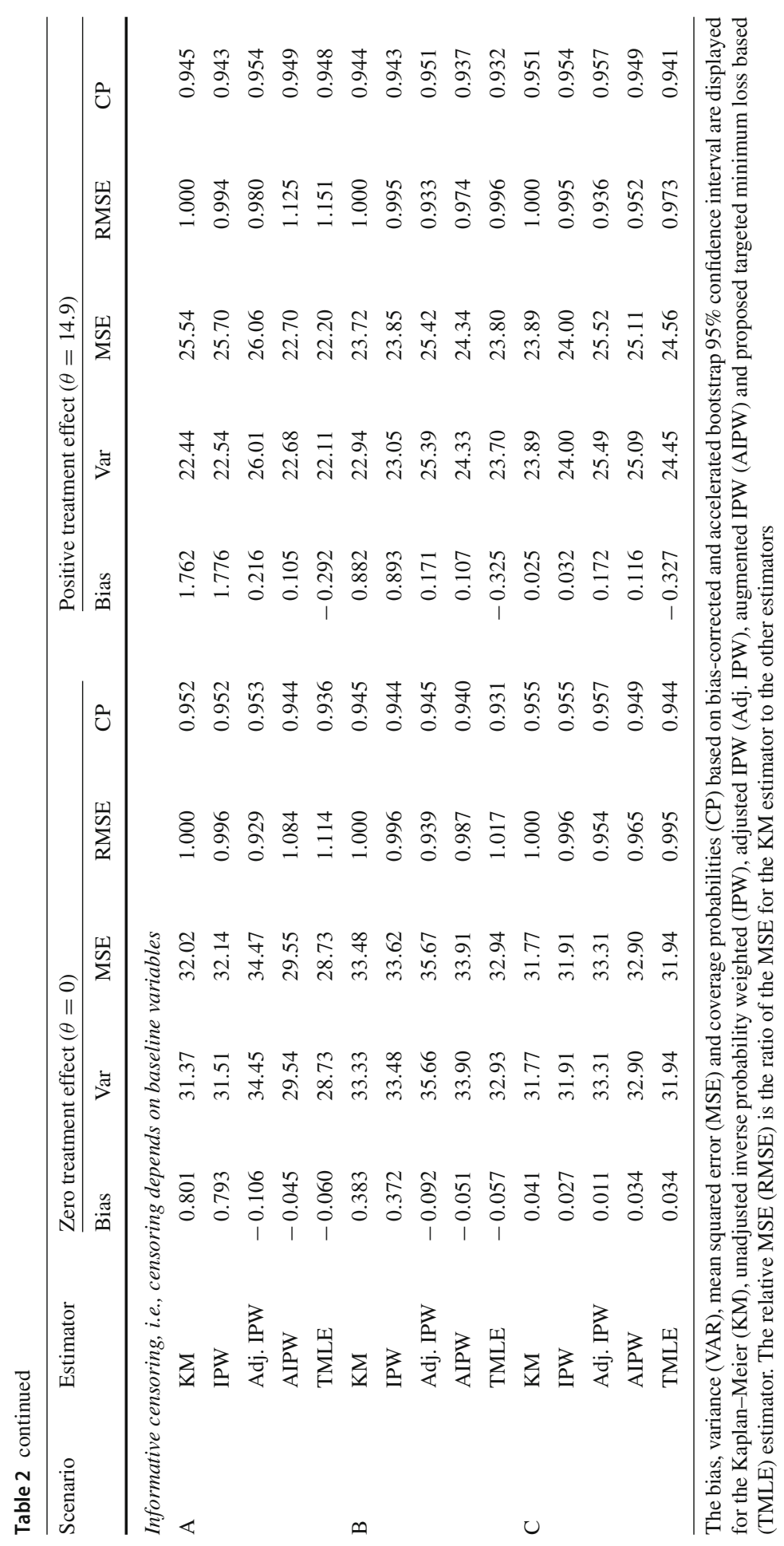




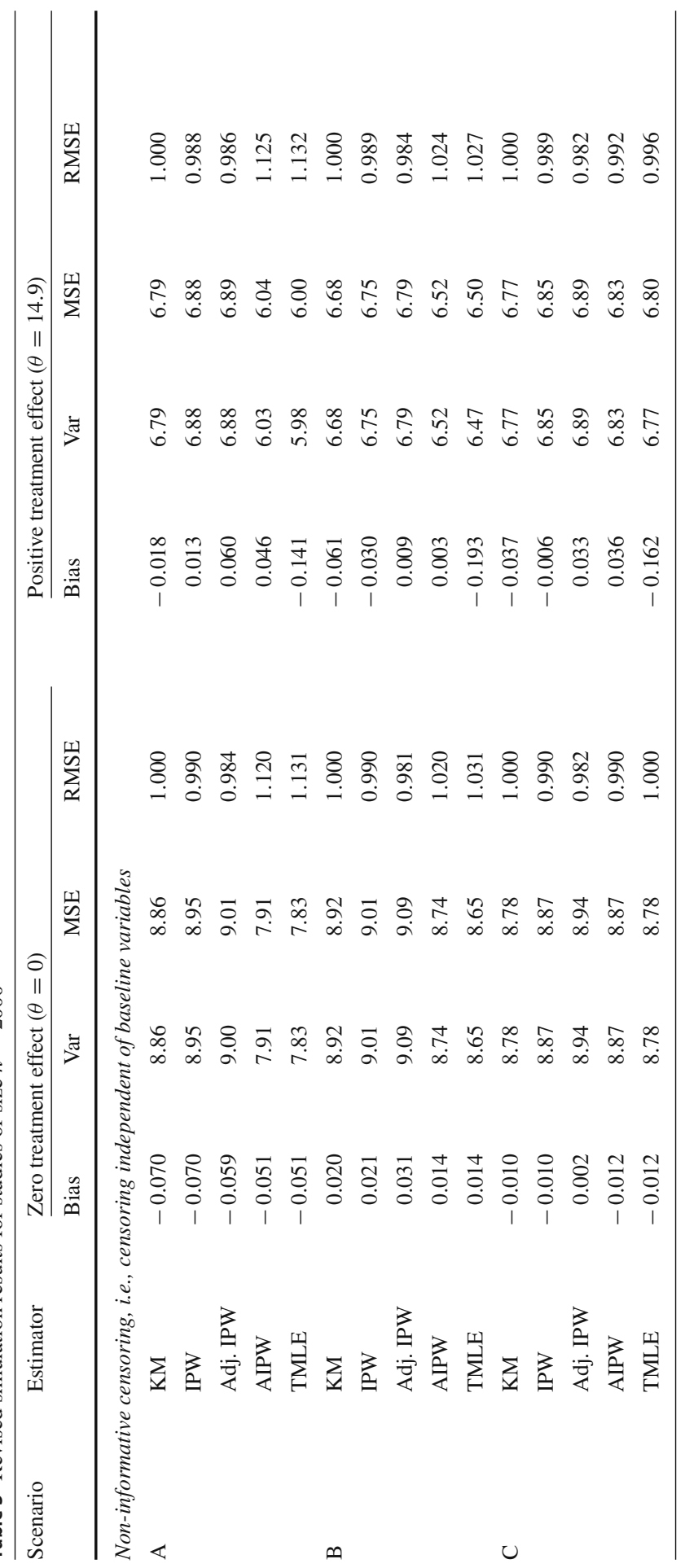




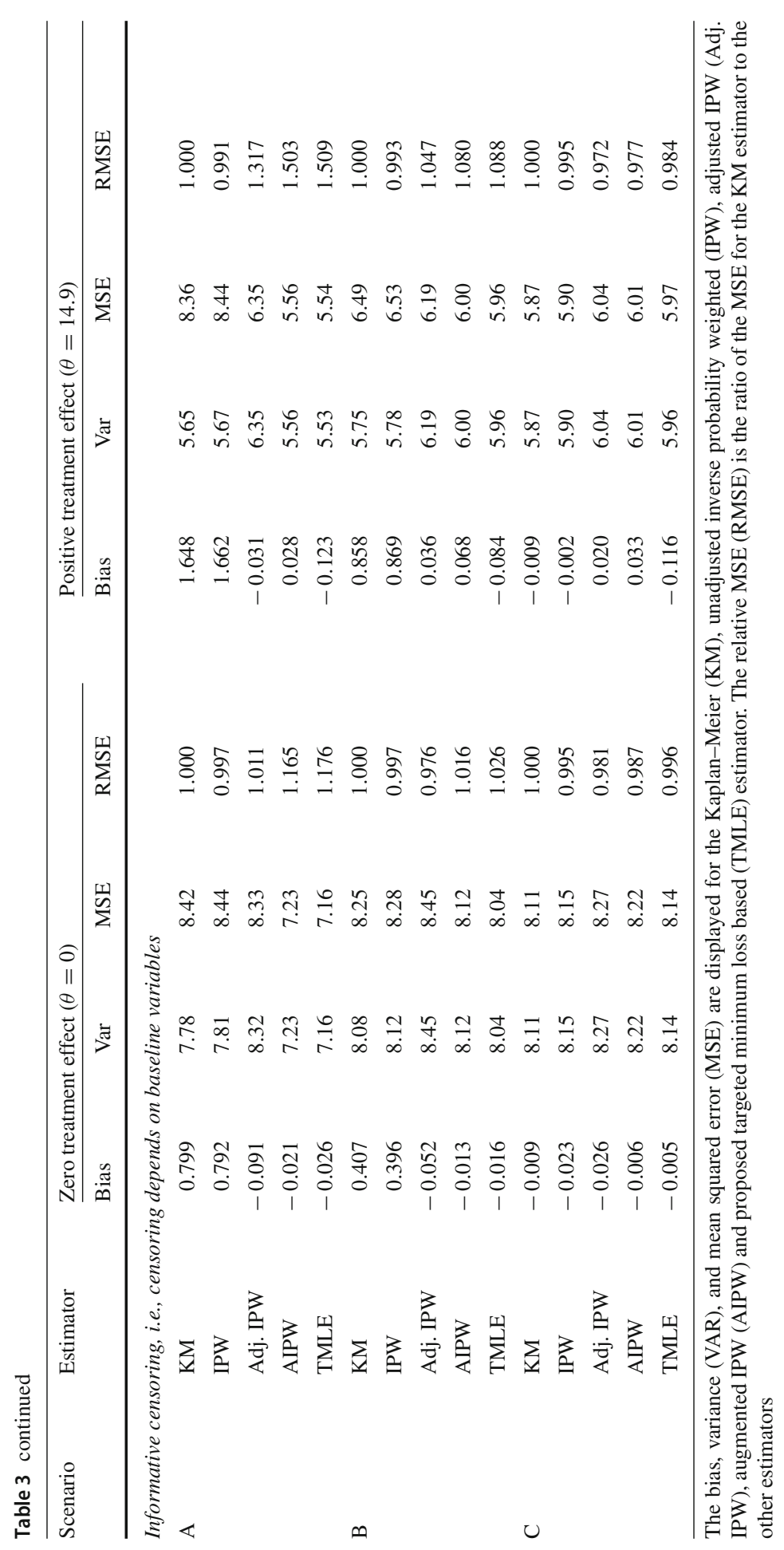


$\left(\hat{\theta}_{\text {adj,eff }}\right)$ confidence interval coverage probabilities ranged from 93 to $95 \%$ (previously 94-95\%); the updated TMLE bias was sometime larger (with maximum absolute value 0.494 in Table 2, top half, positive treatment effect, scenario B), but still was small (at most $3 \%$ ) as a fraction of the treatment effect (14.9 days) and had negligible influence on the mean squared error; the adjusted inverse probability weighted (adjusted IPW) estimator $\hat{\theta}_{\text {adj,ipw }}$ had larger variance.

The most important numerical differences in comparison with our initial results are highlighted in boldface, and all involve the adjusted IPW estimator. The only qualitative change to the conclusions of our initial study is as follows. Inclusion of time as a non-parametric term in our models increased variability in the inverse probability weights of $\hat{\theta}_{\text {adj,ipw }}$. As a result, in most scenarios, this estimator was more variable. Most of the gains in MSE for the adjusted IPW compared to the Kaplan-Meier estimator that were reported in our original manuscript were not present after the errors were fixed.

Acknowledgements We would like to thank Daniel Scharfstein, whose report of the first error in our GitHub code prompted us to write this erratum.

\section{References}

Díaz I (2018a) R package survtmlerct. https://github.com/idiazst/survtmlerct. commit: eb7cd4ea77da21c301b56591180b734c9e305c47

Díaz I (2018b) R package survtmlerct. https://github.com/idiazst/survtmlerct. commit: 9823a4067daf60ebb63f38359ca5569b5df80e0d

Díaz I, Colantuoni E, Hanley DF, Rosenblum M (2018) Improved precision in the analysis of randomized trials with survival outcomes, without assuming proportional hazards. Lifetime data analysis, pp 1-30

Publisher's Note Springer Nature remains neutral with regard to jurisdictional claims in published maps and institutional affiliations. 